\title{
Pinning Force in Superconducting Wires $\mathrm{Nb}-\mathrm{Ti}$
}

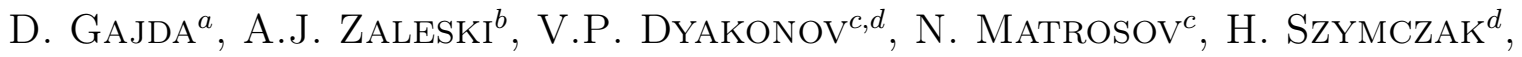 \\ V. Beloshenko ${ }^{c}$, V. SpuskanyuK ${ }^{c}$ And V. Chishro $^{c}$ \\ ${ }^{a}$ International Laboratory of High Magnetic Fields and Low Temperatures \\ Gajowicka 95, 53-421 Wrocław, Poland \\ ${ }^{b}$ Institute of Low Temperature and Structure Research, Polish Academy of Sciences \\ Okólna 2, 50-422 Wrocław, Poland
}

${ }^{c}$ A.A. Galkin Donetsk Physicotechnical Institute, R. Lyuksemburg 72, 83114 Donetsk, Ukraine

${ }^{d}$ Institute of Physics, Polish Academy of Sciences, al. Lotników 32/46, 02-668 Warsaw, Poland

In the paper we present the results of the measurements of critical current $I_{\mathrm{c}}$ in NbTi wires. Experiments were made for the wires of $0.3 \mathrm{~mm}$ in diameter and the length of $25 \mathrm{~mm}$. The wires were produced by means of two methods: conventional (hydroextrusion and drawing) and preliminary equal channel multiple angle pressing. The samples were heat treated at different temperatures. We describe the influence of different pinning centers on critical current density $J_{\mathrm{c}}$.

PACS numbers: 84.71.Mn

\section{Introduction}

The pinning force $F_{\mathrm{p}}$ is a vector product of critical current density $J_{\mathrm{c}}$ and magnetic field $B$. The $F_{\mathrm{p}}$ is very high when pinning centers possess the size similar to coherence length. The coherence length in NbTi is $5 \mathrm{~nm}$. In $\mathrm{NbTi}$ wires the pinning centers are $\alpha$ Ti precipitations. The $F_{\mathrm{p}}$ increases when the distribution of $\alpha \mathrm{Ti}$ precipitations in the whole volume of the sample is homogeneous. The length between $\alpha$ Ti precipitations influences the $F_{\mathrm{p}}$ of NbTi. The size and the shape of $\alpha$ Ti precipitations depend on annealing temperature and annealing time. The long annealing time and high annealing temperature cause the increase of the thickness of $\alpha$ Ti precipitations (200 $\mathrm{nm}$ to $800 \mathrm{~nm}$ ). The pinning force also depends on the crystal dislocation, defects and voids. Technological processes also have impact on the shape of $\alpha$ Ti precipitations.

\section{Samples preparation}

The NbTi wires were obtained in NbTi $60 \% \mathrm{Ti}$ alloy in a copper matrix. In the first stage of the process the NbTi $60 \%$ Ti material underwent hot pressing at $750{ }^{\circ} \mathrm{C}$. Next, the NbTi wires were treated with the two methods: traditional method and equal channel multi-angle pressing (ECMAP) [1]. The traditional method included hydroextrusion (HE) and the process of drawing. The NbTi wires of $3.6 \mathrm{~mm}$ diameter were obtained by means of the HE method. The next step involved drawing NbTi wires. After this process diameters equaled respectively $0.3 \mathrm{~mm}$ and $0.15 \mathrm{~mm}$. The second method involved ECMAP treatment [1]. ECMAP was done by pressing the NbTi wire through a three-angle deforming system $\left(80^{\circ}, 70^{\circ}\right.$ and $\left.80^{\circ}\right)$ of four intersecting channels of equal cross section. The degree of deformation was $e=0.82$ per pass [1]. The samples were obtained in four various passes ECMAP: first, after 4 passes $-e=3.28$, second after 8 passes $e-6.56$, third after 12 passes $e-9.84$, fourth after 16 passes $e-13.12$. After the process of ECMAP NbTi wires were deformed by HE and drawing. The NbTi wires were drawn to diameter $0.3 \mathrm{~mm}$ and $0.15 \mathrm{~mm}$. The NbTi wires contain from $45 \%$ to $50 \%$ of superconducting material. The NbTi wires were annealed at temperatures ranging from $300^{\circ} \mathrm{C}$ to $450^{\circ} \mathrm{C}$.

\section{Experiment, discussion and results}

The critical current $I_{\mathrm{c}}$ of the wires was measured by the four-probe resistive method in liquid helium, constant magnetic field (14 T) and constant current (150 A). The $J_{\mathrm{c}}$ and $F_{\mathrm{p}}$ were obtained with the following formula:

$$
\begin{aligned}
& J_{\mathrm{c}}=\frac{I_{\mathrm{c}}}{S}, \\
& F_{\mathrm{p}}=J_{\mathrm{c}} \times B,
\end{aligned}
$$

where $I_{\mathrm{c}}$ - critical current, $J_{\mathrm{c}}$ - critical current density, $B$ - magnetic field, $F_{\mathrm{p}}$ - pinning force, $S$ - cross-section of the superconducting material.

In the NbTi wires there can be found two pinning mechanisms. The kind of those pinning mechanisms depends on the following factors: pinning centres, density of pinning centres and the arrangement of pinning centres [2].

As far as pinning centres parameters are concerned, they depend on the process of cold drawing, ECMAP, annealing time and temperature. The main objective of our research was to obtain high $F_{\mathrm{p}}$ in a high magnetic 


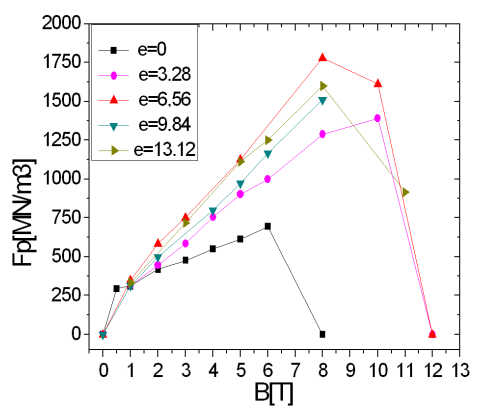

Fig. 1. $\quad F_{\mathrm{p}}-B$ curves for constant annealing temperature $T=400{ }^{\circ} \mathrm{C}$.

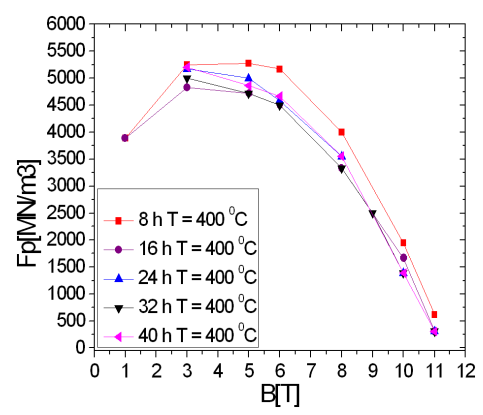

Fig. 2. $\quad F_{\mathrm{p}}-B$ curves for different passes ECMAP.

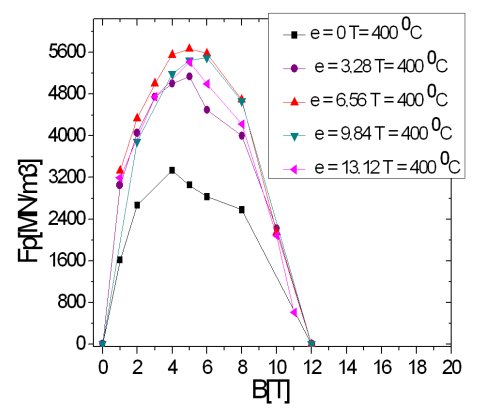

Fig. 3. $\quad F_{\mathrm{p}}-B$ curves for constant annealing temperature and different passes ECMAP.

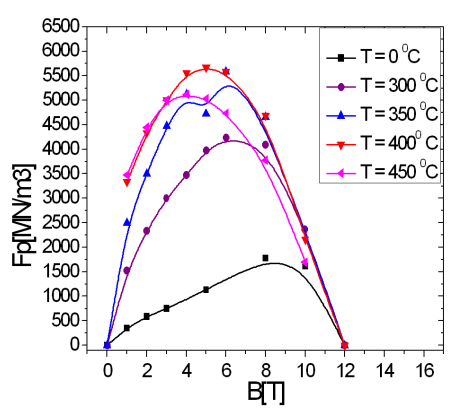

Fig. 4. $F_{\mathrm{p}}-B$ curves for constant passes ECMAP $e=6.54$ and different annealing temperatures.

field. Large density of the pinning centres and their little thickness enabled to achieve maximum $F_{\mathrm{p}}$ in a high magnetic field (Fig. 1 and Fig. 4). Pinning centres with such parameters are obtained by means of cold drawing and ECMAP. Smaller density of the pinning centres and their high thickness allow to gain high $F_{\mathrm{p}}$ but only in smaller magnetic fields. Pinning centres of the same parameters are obtained with the use of annealing time and temperature (Fig. 2 and Fig. 3). When steering the thickness of pinning centres, their density and arrangement by means of the process of annealing and cold drawing, it is possible to obtain maximum $F_{\mathrm{p}}$ in high magnetic fields (Fig. 5a and b).

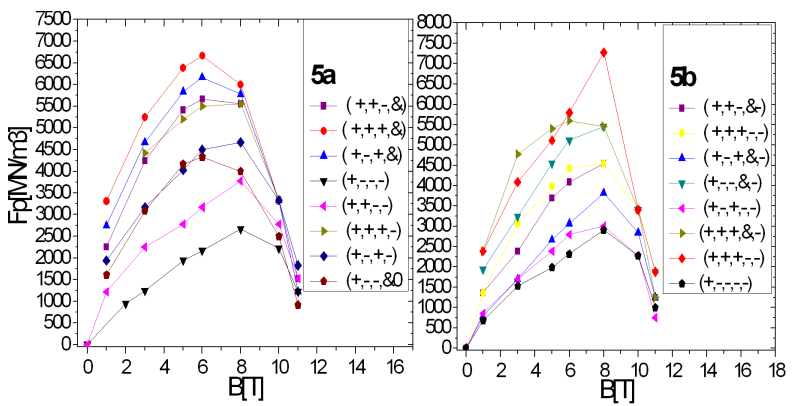

Fig. 5. (a) $F_{\mathrm{p}}-B$ curves for constant annealing temperature, constant passes ECMAP $e=9.84$ and diameter wires $0.3 \mathrm{~mm}$. (b) $F_{\mathrm{p}}-B$ curves for constant annealing temperature, constant passes ECMAP $e=9.84$ and diameter wires $0.15 \mathrm{~mm}$. Each of the following symbols ,,$+- \&$ denotes the number of methods that were used to work the wires: symbol - denotes the lack of annealing temperature, symbol + denotes annealing temperature that equals $400^{\circ} \mathrm{C}$, symbol \& denotes annealing temperature that equals $300^{\circ} \mathrm{C}$.

\section{Conclusion}

The annealing temperature shifts $F_{\text {pmax }}$ to a low magnetic field, because the thickness of $\alpha$ Ti precipitations increases. The process of cold drawing causes the shift of $F_{\text {pmax }}$ to a high magnetic field because the thickness of $\alpha$ Ti precipitations decreases. The research has proven that the new method ECMAP causes the increase of $F_{\mathrm{p}}$ in very high magnetic fields ( $8 \mathrm{~T}$ to $10 \mathrm{~T})$.

\section{Acknowledgments}

This work was partially supported by the grant no. N N508 392035.

\section{References}

[1] V.A. Beloshenko, N.I. Matrosov, V.V. Chishko, O.N. Mironova, É.A. Medvedskaya, D. Gajda, A.J. Zaleski, V.P. Dyakonov, R. Puźniak, H. Szymczak, Low Temp Phys. 34, 606 (2008) (in Russian).

[2] H. Wada, K. Itoh, K. Tachikawa, Y. Yamada, S. Murase, J. Appl. Phys. 57, 4415 (1985). 